\title{
Pacific
}

Journal of

Mathematics

\section{THE FLAT PART OF NON-FLAT ORBIFOLDS}

FENG XU

Volume 172 No. 1 


\title{
THE FLAT PART OF NON-FLAT ORBIFOLDS
}

\author{
FENG XU
}

\begin{abstract}
We use integrable lattice models to determine the complete invariants of a series of new finite depth orbifold subfactors from Hecke algebras.
\end{abstract}

\section{Introduction.}

Commuting squares are an efficient way of producing subfactors. One can always ask the question about how to determine the higher relative commutants once a subfactor is produced. The interest in this question is that in many well-known examples, these higher relative commutants which are finite dimensional $\mathrm{C}^{*}$ algebras if the index is finite, seem to be the right "Quantum symmetry" $([\mathbf{2}])$. In the language of coupling system of Ocneanu([3]), the question is to determine the flat part of a connection. In [1], we found a necessary and sufficient for a certain class of connections from orbifold construction([4]) to be flat. In this paper, we will determine the flat part of those non-flat connections. The exact meaning of this will be explained in Section 1. It turns out that the subfactor constructed from those nonflat connections is the same as a sufactor from a flat orbifold construction, where one does orbifold with respect to a subgroup of the original abelian group. (See the theorem of Section 2.) The paper is organized as follows: In Section 1 we recalled the part of $[\mathbf{1}]$ we need as well as fixing the notations. In Section 2 we proved the main result. This paper is a continuation of [1].

\section{Orbifold Constructions in subfactors.}

The material of this section is contained in Section 2 of [1] and Section 4 of [4]. Let $G$ be a connected, simply connected, compact simple lie group with nontrivial center, i.e. $G=S U(N), S O(2 N+1), S O(2 N), S P(2 N), E_{6}, E_{7}$. Let $Z$ be a nontrivial subgroup of the center $Z(G)$ of $G$. Let $\phi$ be a finite dimensional representation of $G . K \in N$ is a fixed integer(level). In [5], a coupling system associated to $\phi$ is constructed, denoted by $\left(g_{\phi}(K), h_{\phi}(K), B, \tau\right)$. Here $g_{\phi}(K)$ is the principal graph constructed out of the fusion graph of $\phi, h_{\phi}(K)$ is its dual. If $\mathrm{K}$ is such that $Z(0) \in g_{\phi}^{\text {even }} \cap h_{\phi}^{\text {even }}$, since the connection is invariant under the action of the central element (see 2.12 of [1]), one can apply orbifold method with respect to $\mathrm{Z}$ to this coupling system. To get 
a better picture of the orbifold construction, let us take a concrete example, namely, let us describe the orbifold construction of the Wenzl's subfactor as in [4]. It corresponds to the case $G=S U(N), \phi$ is the fundamental representation of $\mathrm{SU}(\mathrm{N}), \mathrm{Z}$ is the cyclic group $Z_{N}$ and $N \mid K$. Let

$$
P_{++}^{n}=\left\{\lambda=\sum_{1 \leq i \leq N-1} \lambda_{i} \Lambda_{i} \mid \lambda_{i} \geq 1, \sum_{1 \leq i \leq N-1} \lambda_{i} \leq n-1\right\}
$$

where the $\Lambda_{i}$ 's are the $N-1$ weights of the fundamental representations and $n=k+N$. For fixed N, we define $\mathcal{A}^{n}$ as follows. The vertices of $\mathcal{A}^{n}$ are given by elements of $P_{++}^{n}$ and its oriented edges are given by $\mathrm{N}$ vectors $e_{i}$ defined by $e_{1}=\Lambda_{1}, e_{i}=\Lambda_{i}-\Lambda_{i-1}, i=1, \ldots, N-1, e_{N}=-\Lambda_{N-1}$. We define an action of the cyclic group $Z_{N}$ as follows. We set $A_{0}=\star$, and label the other end vertices of the graph $\mathcal{A}^{n}$ by $A_{1}=A_{0}+(n-N) e_{1}, A_{2}=A_{1}+(n-N) e_{2}, \ldots$, $A_{N-1}=A_{N-2}+(n-N) e_{N-1}$. Define a rotation symmetry $\rho$ of the graph by $\rho\left(A_{j}+\sum_{k} c_{k} e_{k}\right)=A_{j+1}+\sum_{k} c_{k} e_{k+1}$, where the indices are in $Z / N Z$ and $c_{k} \in C$. Note that $\rho^{N}=i d$. The connection $W$ (see [3]) which is used to embed a small algebra into a big one, is invariant under this action. The vertices of $\mathcal{A}^{n}$ can be colored by $N$ colors in $Z / N Z=0,1, \ldots, N-1$ so that the starting vertex has color 0 and each oriented edges goes from a color $\mathrm{k}$ to a color $\mathrm{k}+1, k \in Z / N Z$. $\mathcal{A}_{r}^{n}$ is A subgraph which has vertices of colors $\mathrm{r}$ and $r+1, r \in Z_{N}$. Let

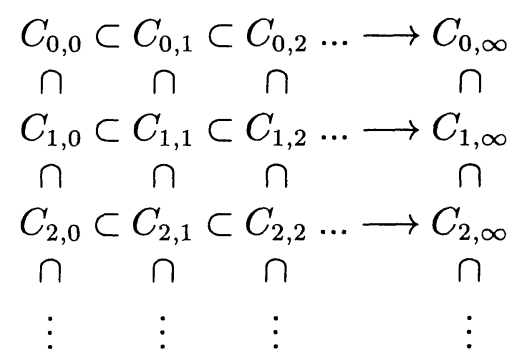

be the double sequences constructed as in [4]. Since the connection is invariant under the action of the center one can apply $\rho$ to each $C_{l, m}$ as a *algebra isomorphism and it is compatible with the inclusions of these algebras. Set $D_{m, n}$ to be the fixed point algebra $C_{m, n}$ of under $\rho$. We get another double string algebras simply replacing $D_{m, n}$ by $C_{m, n}$. The double sequences constructed here are a little different from that of $[\mathbf{3}]$. The source of the string is allowed to be any of the $A_{j} \mathrm{j}=0,1,2 \ldots \mathrm{N}-1$, where in [3] the source is always $A_{0}$. However, the subfactor $C_{0, \infty} \subset C_{1, \infty}$ is the same as the Wezl's subfactor which is the subfactor constructed from similar double sequences but restricts the source to be $A_{0}$. This is also clearly explained in [4]. The reason is the so-called relative Mcduff properties of subfactors constructed out of the commuting squares. If one takes the projection $p$ corresponding 
to $A_{0}$, since $p \in C_{0, \infty}$, the relative Mcduff property implies $C_{0, \infty} \subset C_{1, \infty}$ is isomorphic to $p C_{0, \infty} p \subset p C_{1, \infty} p$ which is the standard description of Wenzl's subfactor in [3]. Set $D_{l, \infty}$ to be the weak closure of $\cup_{m} D_{l, m}$ in its GNSrepresentation with respect to the trace. The subfactor $D_{0, \infty} \subset D_{1, \infty}$ is called orbifold subfactors. By [3], the question of determining the flat part of the orbifold subfactor including finding those $\sigma^{\prime} \in D_{l, 0}$ such that they commute with all $\sigma \in D_{0, m}$. Since by the main result of [1], the connection is flat when $\mathrm{N}$ is odd, but when $\mathrm{N}$ is even, the connection is flat iff $2 N \mid K$. When the connection is flat, the higher relative commutants are simply given by sequences of algebras $D_{m, 0}, m \geq 0$. If the connection is not flat, the higher relative commutants are strictly smaller subalgebras of $D_{m, 0}, m \geq 0$ which commute with all $\sigma \in D_{0, m}$. Hence our question is to determine the higher relative commutants of the orbifold subfactor $D_{0, \infty} \subset D_{1, \infty}$ in the case $\mathrm{N}$ is a divisor of $\mathrm{K}$ but $2 \mathrm{~N}$ is not and $\mathrm{N}$ is even. Since the orbifold connection in this case is not flat, the orbifold subfactor is called a non-flat orbifold and the question is to determine the higher relative commutants of this orbifold subfactor. This is exactly the meaning of the title of this paper.

\section{The higher relative commutants.}

Now we are ready to compute the higher relative commutants. For the sake of completeness, let us first state the theorem in its general form. Let $G$ be a connected, simply connected, compact simple lie group with nontrivial center, i.e. $G=S U(N), S O(2 N+1), S O(2 N), S P(2 N), E_{6}, E_{7}$. Let Z be a nontrivial subgroup of the center $\mathrm{Z}(\mathrm{G})$ of $\mathrm{G}$. Let $\theta_{Z}$ denote the set of fundamental weights of $\mathrm{G}$ associated to $\mathrm{Z}$, and $($,$) the killing form of$ G. In [5], a coupling system associated to $\phi$ is constructed, denoted by $\left(g_{\phi}(K), h_{\phi}(K), B, \tau\right)$. Here $g_{\phi}(K)$ is the principal graph constructed out of the fusion graph of $\phi, h_{\phi}(K)$ is its dual. If $\mathrm{K}$ is such that $Z(0) \in g_{\phi}^{\text {even }} \cap h_{\phi}^{\text {even }}$, since the connection is invariant under the action of the central element (see 2.12 of [1]), one can apply orbifold method with respect to $\mathrm{Z}$ to this coupling system. Let $\phi$ be a finite dimensional representation of G. $K \in N$ is a fixed integer(level).

Theorem. Let $K, Z$ be as before. Let $M$ be the least natural number such that: $1 / 2 M\left(\theta_{z}, \theta_{z}\right) \in \mathbb{Z}, \forall \theta_{z} \in \theta_{Z}$ and let $N$ be the least nature number such that $M \mid N \times K$. Let $Z^{N}$ be the abelian subgroup of $Z$ generated $z^{N}$ for all $z \in Z$. Then the orbifold subfactor constructed out of the action of $Z$ is the same as the orbifold subfactor constructed out of the action of $Z^{N}$ which is necessarily flat (hence the flat part is easy to determine).

As in Section 1, we are going to use orbifold subfactor of Wenzl's subfactor 
to explain and prove the theorem. The proof of the general case is exactly the same except possible change of notations.

Let us assume we are given the conditions at the end of Section 1, namely, $\mathrm{N}$ is a divisor of $\mathrm{K}$ but $2 \mathrm{~N}$ is not and $\mathrm{N}$ is even. As explained in Section 1, this is the only interesting case. In this case, the theorem says the orbifold subfactor with respect to the cyclic group $Z_{N}$ is the same as new orbifold subfactor with respect to the subgroup $Z_{N / 2}$ of $Z_{N}$ which is flat by [1].

Let us first describe this new subfactor in a similar double sequences as that of Section 1.

Let $N=2 N_{1}, \rho$ the action of $Z_{N}$ as in Section 1. Then the $Z_{N / 2}$ action is given by $\rho^{2 m}, \mathrm{~m}=0,1 \ldots N_{1}$. The orbit of distinguished vertex $A_{0}$ under the action of $\rho^{2 m}, \mathrm{~m}=0,1 \ldots N_{1}$ are vertices $A_{2 m}, \mathrm{~m}=0,1 \ldots N_{1}$ Let

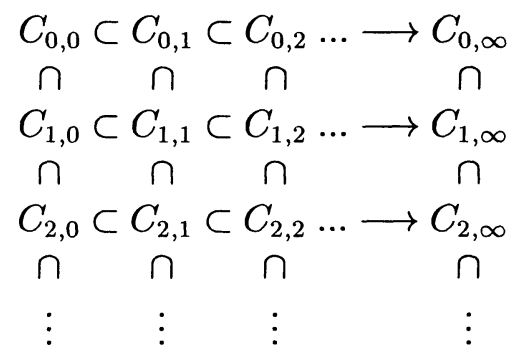

be the double sequences as in Section 1, except that the sources of the strings are restricted to the vertices $A_{2 m}, \mathrm{~m}=0,1 \ldots N_{1}$. Denote by $\hat{D}_{0, \infty} \subset \hat{D}_{1, \infty}$ the orbifold subfactor under the action of $\rho^{2}$. Since the orbifold connection is flat, the higher relative commutants $\hat{D}_{0, \infty}^{\prime} \cap \hat{D}_{k, \infty}$ is given by $\hat{D}_{k, 0}, k \geq 0$. Take paths $\alpha^{\prime}, \beta^{\prime}$ with the same length on the graph $\mathcal{A}_{0}^{n}$ without orientation and with $s\left(\alpha^{\prime}\right)=A_{0}, s\left(\beta^{\prime}\right)=A_{j}$, j is even, $r\left(\alpha^{\prime}\right)=r\left(\beta^{\prime}\right)=C_{0}$, where $C_{0}$ is some vertex of $\mathcal{A}_{0}^{n}$. Let $\sigma^{\prime}=\sum_{l=0}^{N_{1}-1}\left(\rho^{2 l}\left(\alpha^{\prime}\right), \rho^{2 l}\left(\beta^{\prime}\right)\right)$. Note that $\sigma^{\prime \prime}$ s of the above form with the length $\mathrm{k} \operatorname{span} D_{k, 0}^{\prime} k \geq 0$. Similarly another family of higher relative commutants $\hat{D}_{1, \infty}^{\prime} \cap \hat{D}_{k, \infty}, k \geq 1$, are spaned by similar $\sigma^{\prime}$ 's, except that one takes paths on the graph $\mathcal{A}_{N-1}^{n}$ which is dual to $\mathcal{A}_{0}^{n}$. We will show in the following that the higher relative commutants of the orbifold subfactor with respect to the cyclic group $Z_{N}$ are isomorphic to that of $\hat{D}_{0, \infty} \subset \hat{D}_{1, \infty}$ which is of finite depth. Hence they are isomorphic subfactors, thus completing the proof of the theorem.

Let us first determine $D_{0, \infty}^{\prime} \cap D_{k, \infty}$. Since the connection is not flat, the higher relative commutants are strictly smaller subalgebras of $D_{k, 0}, k=0, \overline{1} \ldots$ which commute with all $\sigma \in D_{0, k}, k=0,1 \ldots$. Let $\alpha, \beta$ be paths with the same length on $\mathcal{A}^{n}$ and with $s(\alpha)=A_{0}, s(\beta)=A_{j}, r(\alpha)=r(\beta)=$ $B_{0}$, where $B_{0}$ is some vertex of $\mathcal{A}^{n}$. Set $\sigma=\sum_{l=0}^{N-1}\left(\rho^{l}(\alpha), \rho^{l}(\beta)\right)$. Note that $\sigma$ 's of the above form span $D_{0, k}, k=0,1 \ldots$. Similarly take paths $\alpha^{\prime}$, 
$\beta^{\prime}$ with the same length on the graph $\mathcal{A}_{0}^{n}$ without orientation and with $s\left(\alpha^{\prime}\right)=A_{0}, s\left(\beta^{\prime}\right)=A_{j}, r\left(\alpha^{\prime}\right)=r\left(\beta^{\prime}\right)=C_{0}$, where $C_{0}$ is some vertex of $\mathcal{A}_{0}^{n}$. Let $\sigma^{\prime}\left(\alpha^{\prime}, \beta^{\prime}\right)=\sum_{l=0}^{N-1}\left(\rho^{l}\left(\alpha^{\prime}\right), \rho^{l}\left(\beta^{\prime}\right)\right)$. A general element $\sigma^{\prime} \in D_{k, 0}$ may be expressed as: $\sigma^{\prime}=\sum_{\left|\alpha^{\prime}\right|=\left|\beta^{\prime}\right|=k} \lambda_{\alpha^{\prime}, \beta^{\prime}} \sigma^{\prime}\left(\alpha^{\prime}, \beta^{\prime}\right)$, where $\lambda_{\alpha^{\prime}, \beta^{\prime}}$ 's are complex numbers. We have to study under what conditions we get $\sigma \sigma^{\prime}=\sigma^{\prime} \sigma$. As in [4], it is equivalent to the following :

(1) $\sum_{\eta, \eta^{\prime}, \alpha^{\prime \prime}, \beta^{\prime \prime}, \alpha^{\prime}, \beta^{\prime}, s\left(\beta^{\prime \prime}\right)=s\left(\beta^{\prime}\right)=k}\left|\lambda_{\alpha^{\prime \prime}, \beta^{\prime \prime}} x_{\alpha^{\prime \prime}, \beta^{\prime \prime}, \eta, \eta^{\prime}, l}-\lambda_{\alpha^{\prime}, \beta^{\prime}} y_{\alpha^{\prime}, \beta^{\prime}, \eta, \eta^{\prime}, l}\right|^{2}=0$.

Where $(\alpha, \beta)$ are fixed paths on $\mathcal{A}^{n}, l \in Z_{N}$ and

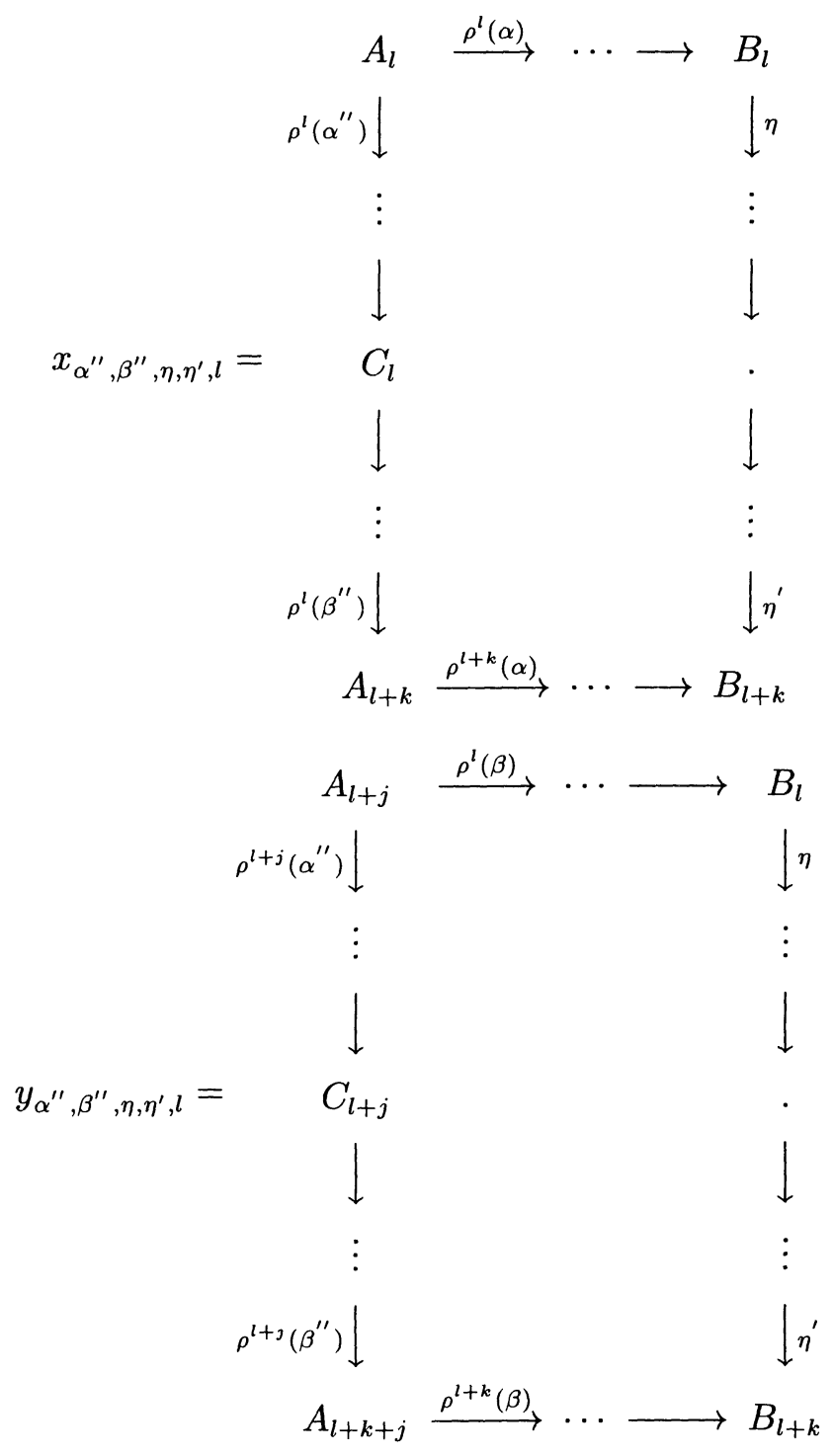


We need the following Orthogonality lemma to simplyfy the above expressions.

Orthogonality Lemma. Let $x_{\alpha^{\prime \prime}, \beta^{\prime \prime}, \eta, \eta^{\prime}}, y_{\alpha, \beta, \eta, \eta^{\prime}}$ be as above. If $\left(\alpha^{\prime \prime}, \beta^{\prime \prime}\right)$ is different from $\left(\alpha^{\prime}, \beta^{\prime}\right)$, Then:

$$
\begin{aligned}
& \sum_{\eta, \eta^{\prime}} x_{\alpha^{\prime \prime}, \beta^{\prime \prime}, \eta, \eta^{\prime}} \bar{y}_{\alpha^{\prime}, \beta^{\prime}, \eta, \eta^{\prime}}=0, \\
& \sum_{\eta, \eta^{\prime}} y_{\alpha^{\prime \prime}, \beta^{\prime \prime}, \eta, \eta^{\prime}} \bar{y}_{\alpha^{\prime}, \beta^{\prime}, \eta, \eta^{\prime}}=0 \\
& \sum_{\eta, \eta^{\prime}} x_{\alpha^{\prime \prime}, \beta^{\prime \prime}, \eta, \eta^{\prime}} \bar{x}_{\alpha^{\prime}, \beta^{\prime}, \eta, \eta^{\prime}}=0 .
\end{aligned}
$$

Proof. We will use the notations from [5]. The lemma follows from the topological invariance. In fact, up to nozero constants, $\sum_{\eta, \eta^{\prime}} x_{\alpha^{\prime \prime}, \beta^{\prime \prime}, \eta, \eta^{\prime}} \bar{y}_{\alpha^{\prime}, \beta^{\prime}, \eta, \eta^{\prime}}$ is equal to the value of the following diagram (see [5] or $[\mathbf{1}]$ ):

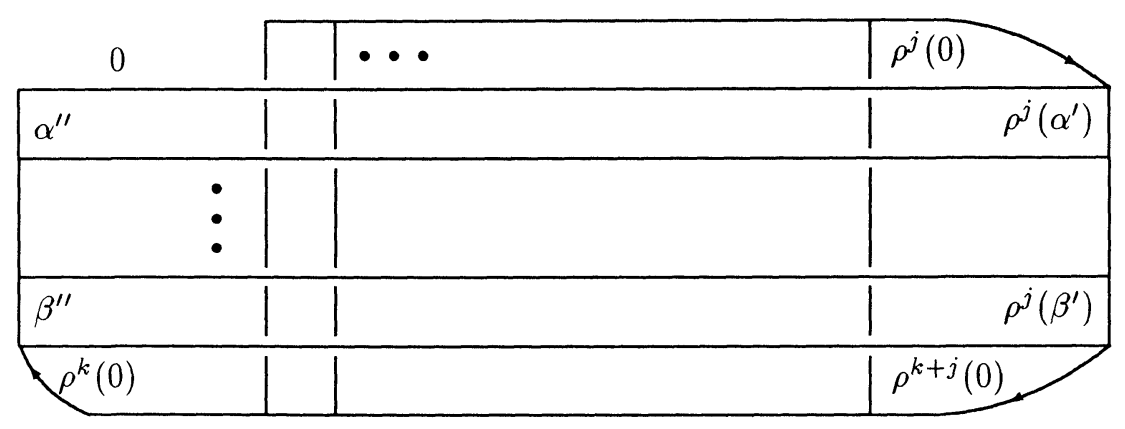

As in [5], the value of the diagram is invariant under regular isotopy, and equal to the value of the diagram:

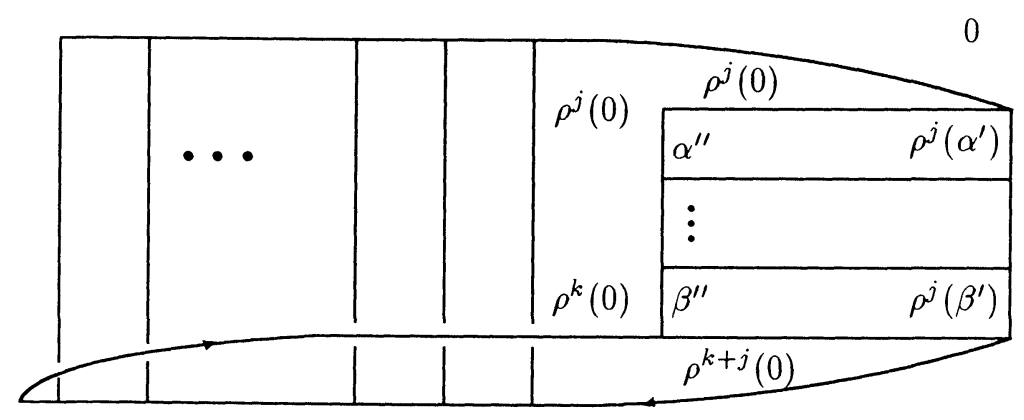


If $\left(\alpha^{\prime \prime}, \beta^{\prime \prime}\right)$ is different from $\left(\alpha^{\prime}, \beta^{\prime}\right)$, the coloring of the above diagram is not admissible, hence the value is 0 . The other two identities follow by the same kind of argument. Q.E.D.

Proof of the theorem. By this lemma, (1) can be simplified to be:

$$
\sum_{\eta, \eta^{\prime}, \alpha^{\prime \prime}, \beta^{\prime \prime}, s\left(\beta^{\prime \prime}\right)=k}\left|\lambda_{\alpha^{\prime \prime}, \beta^{\prime \prime}}\right|^{2}\left|x_{\alpha^{\prime \prime}, \beta^{\prime \prime}, \eta, \eta^{\prime}}-y_{\alpha^{\prime \prime}, \beta^{\prime \prime}, \eta, \eta^{\prime}}\right|^{2}=0 .
$$

By Lemma 2.20 of $[\mathbf{1}]$,

$$
\left|x_{\alpha^{\prime \prime}, \beta^{\prime \prime}, \eta, \eta^{\prime}}-y_{\alpha^{\prime \prime}, \beta^{\prime \prime}, \eta, \eta^{\prime}}\right|^{2}=2-2 R e \exp \left(2 \pi i k j h_{A_{1}}\right) .
$$

Hence (1) is equvialent to : $\exp \left(2 \pi i k j h_{A_{1}}\right)=1$, for all $j \in Z_{N}$ and for all $\left(\alpha^{\prime \prime}, \beta^{\prime \prime}\right)$ such that $\left|\lambda_{\alpha^{\prime \prime}, \beta^{\prime \prime}}\right|$ is not zero, Where $h_{A_{1}}$ denotes the conformal weight of $A_{1}$ (see [5]). Thus $\mathrm{k}$ is even.

To summarize, we have shown that $D_{0, \infty}^{\prime} \cap D_{m, \infty}$ is spaned by: $\sigma^{\prime}\left(\alpha^{\prime}, \beta^{\prime}\right)=$ $\sum_{l=0}^{N-1}\left(\rho^{l}\left(\alpha^{\prime}\right), \rho^{l}\left(\beta^{\prime}\right)\right)$, Here $\alpha^{\prime}, \beta^{\prime}$ are paths with the same length $\mathrm{m}$ on the graph $\mathcal{A}_{0}^{n}$ and the source of $\beta^{\prime}$ is $A_{k}$ with $\mathrm{k}$ even and the source of $\alpha^{\prime}$ is $A_{0}$. These algebras are clearly isomorphic to $\hat{D}_{0, \infty}^{\prime} \cap \hat{D}_{m, \infty}$ as described in the beginning of this section. In fact, let $p$ be the projection in $C_{0, \infty}$ corresponding to those even vertices of the graph $\mathcal{A}_{0}^{n}$, then $p$ commutes with algebras $D_{0, \infty}^{\prime} \cap D_{m, \infty}$ and $p x=0, x \in D_{0, \infty} \cap D_{m, \infty}$ iff $\mathrm{x}=0$. Moreover, $p\left(D_{0, \infty}^{\prime} \cap D_{m, \infty}\right)=\hat{D}_{0, \infty}^{\prime} \cap \hat{D}_{m, \infty}$.

Similarly, one can show that $D_{1, \infty}^{\prime} \cap D_{m, \infty}$ is isomorphic to $\hat{D}_{1, \infty}^{\prime} \cap \hat{D}_{m, \infty}$, where $m \geq 1$. Q.E.D.

We end this section with an example and a remark.

Example. Let $G=S U(2), \mathrm{K}=41+2, \phi$ the spin $1 / 2$ representation. $Z=$ $Z_{2}$. We know that the orbifold is not flat. The theorem says the orbifold subfactor is the same as the original subfactor, that is, the flat part of $D_{n}$ for odd $\mathrm{n}$ is $A_{(2 n-3)}$ subfactor.

Remark. Morally speaking, if one starts with $G / Z$, the question of finding the flat part reduces $G / Z$ to $G / Z^{N}$ which is the correct gauge group as far as the Chern-Simons gauge theory is concerned([6]).

\section{References}

[1] F. Xu, Orbifold construction in subfactors, Comm. Math. Phys., 166 (1994), 237253.

[2] V. Jones, Talk on commuting squares.

[3] A. Ocneanu, Quantum symmetry, differential geometry of finite graphs and classification of subfactors, University of Tokyo Seminary Notes 45 (recorded by Y.Kawahigashi), 1991. 
[4] Y. Kawahigashi and Evans, Orbifold subfactors from Hecke algebra, to appear.

[5] J. de Boer and J. Goeree, Markov traces and $\Pi_{1}$ factors in conformal field theory, Comm. Math. Phys., 139 (1991), 267-304.

[6] E. Witten and Dijigraff, Comm. Math. Phys., 127 (1990), 137-150.

[7] A. Ocneanu, Quantized group string algebras and Galois theory for algebras, London Math. Soc. Lect. Notes Series, 136 (1988).

[8] H. Wenzl, Hecke algebras of type $A_{n}$ and subfactors, Invent. Math., 92 (1988), 345-383.

Received May 15, 1993 and revised December 3, 1993. I came to the question when I heard several interesting talks in V. Jones' seminars given by A. Wasserman to whom I wish to express my gratitude. I'm grateful to my advisor V. Jones for his constant help and encouragement. I also benefit from talks with Y. Kawahigashi.

UNIVERSITY OF CALIFORNIA

Los Angelles, CA 90095

E-mail address: xufeng@math.ucla.edu 



\section{PACIFIC JOURNAL OF MATHEMATICS}

Volume $172 \quad$ No. $1 \quad$ January 1996

A class of incomplete non-positively curved manifolds

BRIAN BOWDITCH

The quasi-linearity problem for $C^{*}$-algebras

41

L. J. BUNCE and JOHN DAVID MAITLAND WRIGHT

Distortion of boundary sets under inner functions. II

Jose Luis Fernandez Perez, Domingo Pestana and José Rodríguez

Irreducible non-dense $A_{1}^{(1)}$-modules

VJACHESLAV M. FUTORNY

$M$-hyperbolic real subsets of complex spaces

101

Giuliana Gigante, Giuseppe Tomassini and Sergio Venturini

Values of Bernoulli polynomials

ANDREW GRANVILLE and ZHI-WEI SUN

The uniqueness of compact cores for 3-manifolds

LUKE HARRIS and PETER SCOTT

Estimation of the number of periodic orbits

BOJU JIANG

Factorization of $p$-completely bounded multilinear maps

Christian Le MERdy

Finitely generated cohomology Hopf algebras and torsion

JAMEs Peicheng LiN

The positive-dimensional fibres of the Prym map

JUAN-CARLOS NARANJO

Entropy of a skew product with a $Z^{2}$-action

KYEWON KOH PARK

Commuting co-commuting squares and finite-dimensional Kac algebras

TAKASHI SANO

Second order ordinary differential equations with fully nonlinear two-point boundary 255 conditions. I

H. BEVAN THOMPSON

Second order ordinary differential equations with fully nonlinear two-point boundary conditions. II

H. BEVAN THOMPSON

The flat part of non-flat orbifolds

FENG XU 